\title{
The Impact of a Comprehensive Medication Counseling and Education on Rehospitalization and Mortality of Advanced Heart Failure Patients in Ghana
}

Charles Anane*, K Ohene-Buabeng, K Sarpong and IK Owusu

Clinical Pharmacist, Cardiology and Critical Care, Ghana

\begin{abstract}
The burden of advanced heart failure includes debilitating symptoms, frequent re-hospitalization and high rates of mortality. The most common causes of this preventable rehospitalization are patient's failure to adhere to prescribed medicines and diet regimen. It is imperative that any medication counseling and educational program seeks to assess the patients understanding of their medical conditions and ability to appreciate the importance of medication compliance to their condition. A prospective cohort study involving 583 patients aged $\geq 30$ years who met clinical criteria for presence of advanced heart failure on admission was done to assess the outcome of the program on mortality and re-hospitalization. 497 patients were discharge to the cardiac clinic for a 6-month follow up during which period they were taken through medication counseling and education on; Medication Compliance, Smoking, Alcohol, Diet and Nutrition, Salt restriction, Fluid intake, Weight loss and Exercise. The study showed $97.6 \%$ decrease in re-hospitalization rate and $12.4 \%$ decrease in mortality. Patients had also significantly improved in subjective and objective indices to functional status. $93.8 \%$ were classified within NYHA function class II and $6.2 \%$ in function class III. None of the patients remained in functional class IV as compared to $46.5 \%$ of the patients before discharge. The study showed that management of heart failure patients based on well structured medication education and counseling modalities can contribute to improved patients outcomes, including reduced morbidity and mortality rates, improved functional status and quality of life.
\end{abstract}

Keywords: Advanced Heart failure; Re-hospitalization; Quality of life; NYHA functional class

\section{Introduction}

The burden of advanced heart failure includes debilitating symptoms, frequent rehospitalization and high rates of mortality. The most common causes of this preventable rehospitalization are patients' failure to adhere to prescribed medicines and diet regimen. It is imperative that any medication counseling and educational program seeks to assess the patients' understanding of their medical conditions and ability to appreciate the importance of medication compliance to their condition [1].

\section{Methodology}

A prospective cohort study involving 583 patients aged $\geq 30$ years who met clinical criteria for presence of advanced heart failure on admission was done to assess the outcome of the program on mortality and re- hospitalization. 498 patients were discharge to the cardiac clinic for a 6-month follow up. These patients were taken through medication counselling and education on the management of their disease condition as follows:

\section{Medication compliance}

Compliance is often seen as one of the most important outcome of patients' education. Therefore, all the patients were counselled and educated to comply with the prescribed treatment and to adapt to health-promoting behaviors like healthy diet and moderate or mild exercise depending on age. The underlying philosophy of compliance was that the illness could be controlled if the patient complied with prescribed therapy. They were taught that non-compliance increases morbidity and mortality and rehospitalization [2].

Medications: The patients were educated on the name of each medicine and its purpose, dosage, frequency and significant side- effects likely to be encountered. For example, patients who were taking $\beta$-blockers were counselled that they may experience fatigue, lightheadedness or dizziness. They were further instructed that their $\beta$-blockers would be up-titrated slowly as tolerated, that sideeffects would minimize over time and that they should report if they experienced any adverse effects. In addition, the patients were instructed to bring all their medications with them to each clinic visit. That served two purposes; first, information about each drug could be reviewed and the patients' knowledge assessed. Secondly, confusion about medicine doses and interactions could be addressed.

In addition, patients were asked about over-the-counter and alternative medications example homeopathic or herbal products they might be taking. They were advised to avoid such products so long as they remained on their cardiac drugs.

Patients were counselled to avoid taking non-steroidal antiinflammatory drugs not prescribed because they could lead to renal dysfunction and renal failure.

Smoking: All the patients were strongly discouraged from cigarette smoking if they were smokers.

*Corresponding author: Charles Anane, Clinical Pharmacist, Cardiology and Critical Care, Medicine Directorate, Head, Clinical Pharmacy, Komfo Anokye Teaching Hospital, Ghana, Tel: 233-1313803; E-mail: chaanane20005@yahoo.com

Received July 10, 2013; Accepted September 5, 2013; Published September 12, 2013

Citation: Anane C, Buabeng KO, Sarpong K, Owusu IK (2013) The Impact of a Comprehensive Medication Counseling and Education on Rehospitalization and Mortality of Advanced Heart Failure Patients in Ghana. J Cardiovasc Dis Diagn 1: 123. doi:10.4172/2329-9517.1000123

Copyright: (C) 2013 Anane C. This is an open-access article distributed under the terms of the Creative Commons Attribution License, which permits unrestricted use, distribution, and reproduction in any medium, provided the original author and source are credited. 
Alcohol: All the patients were counselled to restrict alcohol consumption to moderate levels given the myocardial depressant properties of alcohol.

Diet and nutrition: All the patients were taken through diet and nutrition lessons by the dieticians at the hospital and made to understand that diet and nutritional measures were as important in heart failure to ensure adequate and appropriate nutritional balance [3]. Poor nutrition could contribute to cardiac cachexia. They were advised by the dieticians at the hospital as follows; to avoid commonly consumed processed foods that have high sodium content such as cheese, sausages, salted peanuts, tinned meat e.g. corned beef and tinned fish e.g. sardines, salmon and tuna.

Salt restriction: The patients were educated to restrict their salt intake substantially. All the patients were counselled to avoid foods that are rich in salt and were told not to add salt to their food at the dining table. They were advised to take fresh produce such as fruits, vegetables, eggs and fish which have relatively low salt content.

Fluid intake: Patients with severe symptoms, those requiring high dose diuretics and those with a tendency towards excessive fluid intake were counselled to restrict their fluid intake to 1.5-2 litres daily.

Weight loss: Obese patients were taken through a comprehensive program with the nutritionist to reduce weight.

Exercise: All the patients in stable condition were encouraged to take to light exercising like walking as regular as possible. Although bed rest may be appropriate in patients with acute heart failure, regular exercise was encouraged. Nevertheless, the patients were advised to know their limits and avoid excessive fatigue or breathlessness during mild or moderate exercise $[4,5]$.

Data processing and analysis: The data collected using the Data Collection Forms were coded, transferred to Microsoft office Excel and then entered separately into STATA version 11 statistical software for analysis. Descriptive statistics were used to characterize the study patients. Data were presented as means \pm Standard Deviations (SDs) or frequencies and percentages. The baseline characteristics of the three treatment interventions were compared using t-test and chi-square statistics.

\section{Results}

The patients recorded $100 \%$ in attendance during the $1^{\text {st }}$ three months and $96.6 \%, 95.4 \%$ and $94.3 \%$ attendance during the 4 th, 5th and 6 th months respectively when 12 patients were re-hospitalized (Table 1). However, the table shows in addition the percentages of patients who did not attend the clinic in those months i.e. $1.3 \%, 2.5 \%$ and $3.6 \%$ during the $4^{\text {th }}, 5^{\text {th }}$ and $6^{\text {th }}$ months respectively. During the first 3 months of follow-up, patients scored $100 \%$ in terms of regular attendance, knowledge and compliance of medications and followed all the instructions given them on diet, salt restriction, fluid restriction and the others as showed on the table.

\section{NYHA functional status}

Out of the 498 patients discharge to the cardiac clinic for the followup program, 12 patients were re-admitted and 6 died. The remaining 492 patients completed the full 6-month follow-up counseling and education program at the cardiac clinic. At the end of study period, functional status of the patients had improved from $92.1 \%$ in NYHA class II to $97.1 \%$ in NYHA class II. Only $2.9 \%$ had remained in NYHA class III as compared to $7.9 \%$ before the program. The $2.9 \%$ of the patients who remained in Class III at the end of the study period needed some form of device intervention or valve repairs i.e. pacemakers and mitral valve repair or replacement.

Out of the 583 patients recruited for the study, 14.6\% ( 85 patients) had died before discharge to the cardiac clinic and $85.4 \%$ (498) surviving patients began the the 6-month follow-up program at the cardiac clinic. Out of the 498 patients, 1.2\% (6) patients died during the follow-up with $98.8 \%$ (492) patients remaining alive till the end of the study program (Figure 1).

Out of the 186 patients in the carvedilol group discharged to the cardiac clinic 2 patients had died during the follow-up period and 184 patients in this group were alive till the end of the study period. Out of the 207 patients in the bisoprolol group discharge to the cardiac clinic 1 patient died and 206 patients were alive till the end of the study period. With the control group out of 105 patients discharge to the cardiac clinic, 3 patients had died leaving 102 patients alive till the end of the study period. Mortality difference between the 2 intervention groups did not reach statistical significance but compared to the control group the mortality difference was significant statistically.

Out of the 498 patients discharge to the cardiac clinic, $2.4 \%(\mathrm{n}=12)$ of patients were rehospitalized and 6 died, however, the remaining 492 patients did not need any readmission till the end of the study period (Table 2 and Figure 2).

\section{Knowledge level of the patients}

Almost $80 \%$ of the patients were aware of the importance to limit

\begin{tabular}{|l|l|l|l|l|l|l|}
\hline $\begin{array}{l}\text { Counselling \& } \\
\text { education }\end{array}$ & $\begin{array}{l}\text { 1st } \\
\text { month }\end{array}$ & $\begin{array}{l}\text { 2nd } \\
\text { month }\end{array}$ & $\begin{array}{l}\text { 3rd } \\
\text { month }\end{array}$ & 4th month & 5th month & 6th month \\
\hline Medication & Yes & Yes & Yes & Yes & Yes & Yes \\
\hline Compliance & Yes & Yes & Yes & Yes & Yes & Yes \\
\hline Diet \& nutrition & Yes & Yes & Yes & Yes & Yes & Yes \\
\hline Salt restriction & Yes & Yes & Yes & Yes & Yes & Yes \\
\hline Fluid restriction & Yes & Yes & Yes & Yes & Yes & Yes \\
\hline Weight loss & Yes & Yes & Yes & Yes & Yes & Yes \\
\hline Smoking & Yes & Yes & Yes & Yes & Yes & Yes \\
\hline Alcohol & Yes & Yes & Yes & Yes & Yes & Yes \\
\hline Exercise & Yes & Yes & Yes & Yes & Yes & Yes \\
\hline$\%$ Attendance & 100 & 100 & 100 & 96.6 & 95.4 & 94.3 \\
\hline
\end{tabular}

Table 1: Percentage attendance of the patients at the cardiac clinic and counseling and education sessions

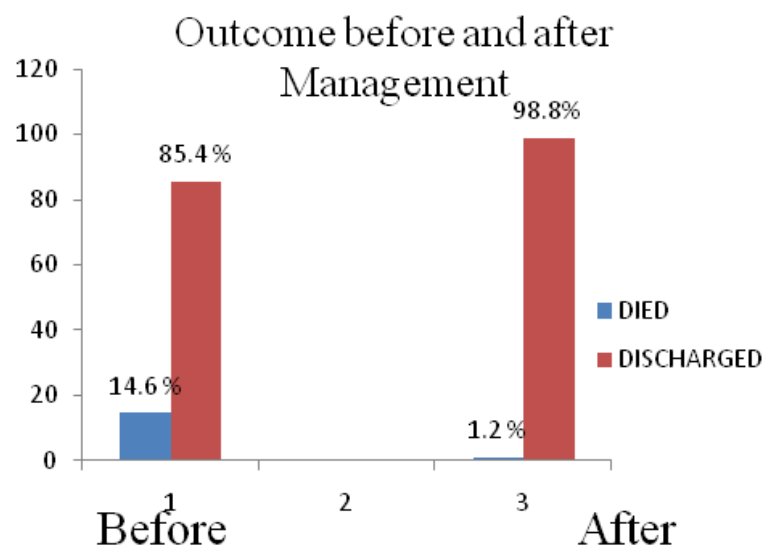

Figure 1: Outcome before and after discharge to the cardiac clinic. 
their salt intake.

\section{Barriers of learning}

Because majority of the patients had little or no formal education, counselling sessions were time-consuming and most of the processes needed repetition over again for the patients to understand and appreciate their importance to their disease progression (Table 3).

\section{Discussion}

$37 \%$ of the cohort had no formal education and these patients took a lot time to understand their disease and medication. With $42 \%$ having just basic education to some level, their understanding of their condition was almost the same with those who had no formal educational group. Patients with tertiary education (7\%) and secondary education (21) were better in their understanding of the disease prognosis and its management.

The goals of the medication counselling and education program were to assist patients to comply with the therapeutic regimen, to maintain clinical stability and function, and to improve quality of life. These goals are best achieved when the patient and family are knowledgeable about every aspect of the condition and treatment and are active participants in the plan of care. With majority of the patients having little knowledge about their condition because of illiteracy, education and counselling was simplified to their understanding and appreciation. Furthermore, since the intervention lasted only 6

\begin{tabular}{|c|c|c|}
\hline NYHA & Before Management & 6 months after Management \\
\hline II & 92.1 & 97.1 \\
\hline III & 7.9 & 2.9 \\
\hline IV & - & - \\
\hline
\end{tabular}

Table 2: Improvement in functional status after 6 months of the counseling program for all the 492 patients that remained alive till the end of study compared with their composite functional status before undergoing the management program irrespective of intervention.

\section{Rehospitalization}

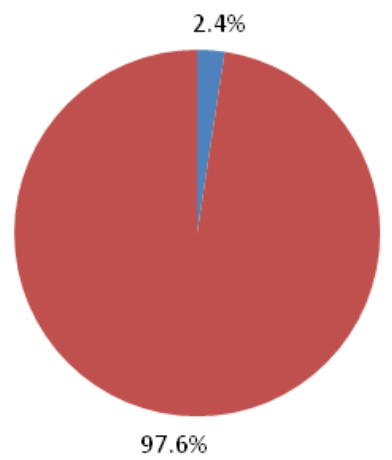

Figure 2: Percentage of the patients who were readmitted after discharge to the cardiac clinic for counseling and education program.

\begin{tabular}{|c|c|}
\hline Education level & $\%$ \\
\hline Tertiary Education & 7 \\
\hline Secondary Education & 21 \\
\hline Primary Education & 42 \\
\hline No Formal Education & 37 \\
\hline
\end{tabular}

Table 3: Literacy levels of the patients months, the optimal length of education and support through other methods like home visits and tele-monitoring is unknown, and the minimum time period necessary for patients to manifest benefits of these interventions is still unknown. The study has showed that an integrated approach to the management of heart failure based on well structured medication education and counselling can contribute to improved patients outcomes, including reduced morbidity and mortality rates, improved functional status and quality of life. The program also enhanced compliance, reduced rates of rehospitalization and prolonged survival. These results, building on the work of others, suggest that all patients with advanced heart failure should be offered an education and support program that extends beyond hospitalization [3-5].

\section{Counselling of the patients}

It was important to explain to the patients the results he/she should expect from adding the $\beta$-blocker to their conventional therapy and that clinical improvement was gradual and may only become evident after treatment for some months [2].

They were further instructed that the $B$-blocker would be uptitrated slowly, as tolerated; that side effects would decrease over time; and that they should call the healthcare team if they experienced any adverse effects.

Patients were encouraged to measure their blood pressure and heart rate frequently and report to the clinic if these values fall outside the optimal range and/or they develop symptoms particularly breathlessness, fatigue and dizziness.

Patients were taught to understand some level of their condition to influence compliance and reduce readmission rate.

These counselling points were very useful as they reflected in the outcome at the end of the program with less patients being readmitted and in mortality reduction.

\section{Conclusion}

The study also showed that an integrated approach to the management of heart failure based on well structured medication education and counselling can contribute to improved patients outcomes, including reduced morbidity and mortality rates, improved functional status and quality of life.

\section{References}

1. Strömberg A, Martensson J, Fridlund B, Levin LA, Karlsson JE, et al. (2003) Nurse-led heart failure clinics improve survival and self-care behaviour in patients with heart failure: results from a prospective, randomized study. Eur Heart J 24:1014-1023.

2. Burkhart PV, Sabaté E (2003) Adherence to long-term therapies: Evidence of Action. J Nurs Scholarsh 35: 207.

3. Vinson JM, Rich MW, Sperry JC, Shah AS, McNamara T (1990) Early readmission of elderly patients with congestive heart failure. J Am Geriatr Soc 38: $1290-1295$.

4. Bennett SJ, Huster GA, Baker SL, Milgrom LB, Kirchgassner A, et al (1998) Characterization of the precipitants of hospitalization for heart failure decompensation. Am J Crit Care 7: 168-174.

5. Ghali JK, Kadakia S, Cooper R, Ferlinz J (1988) Precipitating factors leading to decompensation of heart failure: traits among urban blacks. Arch Intern Med 148: 2013-2016 